\title{
A Link-Stability Aware Routing for improving DPDR in Mobile Ad-hoc Network
}

\author{
Deepthy Mathews \\ PG Scholar \\ Department of Computer \\ Science \& Engineering \\ SNS College of Technology, \\ Coimbatore
}

\author{
S Kannan \\ Assistant Professor \\ Department of Computer \\ Science \& Engineering \\ SNS College of Technology, \\ Coimbatore
}

\author{
S Karthik, PhD. \\ Dean \\ Department of Computer \\ Science \& Engineering \\ SNS College of Technology, \\ Coimbatore
}

\begin{abstract}
Link Stability is a crucial factor in designing routing protocols for Mobile networks. Most of the routing strategies that supports for link stability are designed to meet some QoS constraints. Dynamic nature of nodes and the presence of stale information make this very difficult. In this work, we present a performance evaluation of some of the existing link stability based routing mechanisms and proposes a routing protocol called Link Stability Aware Routing (LSAR) protocol that determines a path with maximum stability. The mechanism uses the concept of both Remaining Link Lifetime and Stability metrics to measure the stability of links in the network. The route with its links having maximum link ages contributes higher Remaining Link Lifetime and thereby considerable path stability. This route is selected for data transfer.
\end{abstract}

\section{Keywords}

MANET, Link Stability, Remaining Link Life time, LSAR, Link Period.

\section{INTRODUCTION}

A-hoc network, as its name indicates is a collection of nodes that are connected arbitrarily for some temporary time without the aid of any fixed infrastructure. Mobile Ad-Hoc Network (MANET) is a type of wireless ad-hoc network which differs from other networks in terms of node mobility and its autonomous operation. Each and every node can act as a host as well as a router. These networks exhibits the features like dynamic topology, bandwidth constrained operation, variable capacity links, energy constrained operation and vulnerable to attack.

MANET has the features such as ease of deployment, lack of infrastructure, auto configuration, de-centralized operation etc which makes it more suitable to be used in application areas like military battle fields, smart offices, emergency rescue operations etc. Even though wide deployment of MANET is still a dream due it's challenges [14] like routing [7], shared wireless medium, resource constrained applications, highly dynamic topology security, QoS, Energy consumption etc among which Qos [13] is an important one. QoS is very much dependent on the stability of network. The mobility of nodes results in frequent path breaks which in turn affects the path stability. Therefore, MANET routing protocols must concentrate not only on shortest distance path, but also on the factors like node mobility, signal strength and interference in the channel.

Link stability is one of the most important factors that affects the quality of service and also accounts for finding a better route in terms of network stability. This is how, when any link corresponding to a path breaks, the path has to be either repaired or an alternative path has to be found out. This rerouting results in energy consumption and delay in data transfer. Also frequent changes in route affect the QoS in the communication by introducing jitter and out of order packet delivery. Thus it is necessary to find a route with maximum stability.

Link stability can be said as the measure of capacity of the links for how much time that link can withstand in that network without any breakage. One of the factors that contribute to path stability is Route Expiration Time (RET) during which all the links along that path will be available. Most of the research works on link stability are based upon the following three factors; mobility of the node, energy of the node and signal strength.

For link stability based routing, a path between source and destination nodes are said to be stable only if it consists of the most stable neighbors at intermediate hops. For Mobile AdHoc network, its features such as node mobility, decentralized operation, fading effects, interference etc causes frequent link breakages and result in an error prone network. So, the protocol must be designed in such a way that it selects a more stable path for data transfer. In this paper, we present a simple and efficient method to select stable wireless links based on the value of link period and remaining link life time values between nodes.

The remaining part of this paper is organized as follows:-In section II, we will discuss the related work done in the field of link stability based routing mechanism. Section III deals with the problem statement and in section IV we introduce our proposed idea for link stability based routing in MANET. In section $\mathrm{V}$, we will do the comparative study of the protocols GPSR and PERRA with our new proposed protocol LSAR. Finally, we will conclude the paper and give the future scope of this paper.

\section{RELATED WORKS}

The aim of stability based routing algorithms is to choose the routes which are more stable at time. Some of the previous works established that by choosing routes based on the parameters like position of the nodes, their remaining battery 
level and mobility patterns will give way for new routing strategies that promising a better and error resilient paths. Such stability oriented routing works consider the link stability as the fundamental criteria.

There are many protocols that concentrate on metrics like link and path stability. Some of them are based on route breakage probability and some others on link duration distribution. But, most of these protocols are by considering some of the parameters connected with mobility models for calculating the stability metric.

In [1], [15] the authors use a statistical prediction method based on the movement of nodes in the network. By using a random mobility model, link stability probability has been defined for finding out a stable route. In [10] the authors proposed a model which predict the lifetime of routes based on a prediction technique and concept of random walk mobility. In this approach, the probability is defined through dividing the area of node movement into different sub cells of and the movement of nodes is observed on that cells.

A state based movement of nodes in the cells is considered and transition probabilities are estimated. Here, the wireless link is considered as a connection between each node in a cell with its neighbor nodes in other cells. The wireless link dynamic between a node and its neighbor nodes is used to calculate the link lifetime. Also, the breakage probability is estimated with the assumption of independent route breakage. There are many papers which uses the concept of signal stability to estimate the link stability probability [2]. The drawback of this technique is that in most cases it is not suitable since signal stability can be affected by some of the environmental conditions. Another reason is that the value of signal strength changes frequently for same set of nodes. Thus there is the chance of variations in radio signal measurements and which in turn affects the link stability.

In [9],[12] the authors proposed a novel approach to determine the residual link lifetime based upon the link age and the previous link measurements. Different metrics were proposed in which one is by selecting youngest link as more stable link since they are more breakage resilient. Another one technique selects the oldest link as stable one. One approach shown is that selecting links with highest residual lifetime yields a stable path. Another metric taken into consideration was the persistence probability. The last one is the connection failure probability which is the robust one.

Another approach is to rely on some devices like Global Positioning System (GPS) to for calculating the correct location of mobile nodes. According to this technique, each node calculates its position and it requests for others position information using some protocols [11]. The main problem of this approach was its difficulty in indoor applications or it is not that much functional when the mobile nodes have limited energy.

In [6] a prediction location based routing approach is introduced to increase the packet delivery ratio of GPSR by selecting more stable path. In [8] the number of route transition a routing protocol incurs in sending data is taken into account to find the path stability. In this work, the concept called Stability delay tradeoff was introduced as a measure of route stability. A protocol called Power Efficient Reliable Routing protocol for Mobile Ad-hoc networks [4] determines a stable path by considering the path stability along with energy metrics calculation.

\section{PROBLEM STATEMENT}

In MANET, the nodes are connected by means of wireless links that varies in the network due to the changes in node's position, mobility and congestion. This variation results in the data packet rate variations and affects the link quality. If the variability is very high, it may result in higher jitter that violates the QoS requirements.

It is evident that, the variations in link quality in turn affect the route quality. So, a route consisting of too many variable links does not result in effective data transfer. Thus, finding out a path which accounts for better link stability and path stability also that requires less control overhead and minimum energy is an important challenge of routing in mobile networks. In order to overcome such a problem, we propose a link stability based routing protocol called LSAR for MANET. The system calculates the link period and the Remaining Link Life time. These stability metrics are used to determine more stable links. Finally, a stable route is selected for the routing purpose.

Table 1. Table captions should be placed above the table

\begin{tabular}{|c|c|c|c|}
\hline Graphics & Top & In-between & Bottom \\
\hline Tables & End & Last & First \\
\hline Figures & Good & Similar & Very well \\
\hline
\end{tabular}

\section{LINK STABILITY AWARE ROUTING PROTOCOL}

In this paper, link stability taken into consideration rather than considering path stability. For that purpose we introduce a concept of link period which is the time duration between the initialization and breakage of that link and which is represented by $p$. For any link from node $i$ to node $j$, its link period is given by,

$$
P(i, j)=t_{\text {final }}-t_{\text {initial }}
$$

Different from other existing techniques a statistical based approach is used to differentiate the stable links from others. A link is said to be stable if it can withstand some amount of time. Based on the observed values of each links in the previous instants, the remaining link lifetime is estimated which is the remaining time that the link can exist without breaking. Here, the remaining link lifetime is essential since stabile links are determined in terms of the probability of that links to stick with the network. The remaining lifetime $R L T_{i, j}\left(p_{i, j}\right)$ for any link from node $i$ to node $j$ with link period $\mathrm{p}_{i, j}$ is determined as,

$$
R L T_{i, j}\left(p_{i, j}\right)=\frac{\sum_{p=p i j}^{P \max } p \cdot a[p]}{\sum_{p=p i j}^{P \max } a[p]}-p_{i, j} \forall(i, j) \in P
$$

where, $P_{\max }$ is the maximum observed period of links, $P$ is the set of links in the network and $a[]$ represents an array used to store all the link period values. The term $a[p]$ represents the count of all the links having link period as $p$. 
Here comes a problem that a stable link cannot be resolved when there are more links with same link period. In order to avoid this drawback, the average of distances $D_{\text {avg }}$ traveled by a node is calculated. This is how, the total distance crossed by the node is stored and its average is estimated. Thus even if there are more than one links with same remaining link lifetime, a path with shorter average distance is taken for transmission.

Using the average distance $D_{\text {avg }}$, a coefficient for stability $S_{i, j}(t)$ if calculated using equation,

$$
S_{i, j}(t)=\frac{\text { Davg }}{R L T i_{i} j(p i, j)} \forall\left(i_{i} j\right) \in P .
$$

This stability coefficient can be considered as the reciprocal of stability. This is how as the Remaining link lifetime increases, the link will be more stable. Also, for higher the average traveled distance, the chance for link breakage also is higher.

\section{RESULTS AND DISCUSSIONS}

In order to evaluate the LSAR protocol, NS-2 Simulator [3] is used. Some comparisons are made with other two protocols PERRA and GPSR. It is shown that LSAR performs well in terms of data packet delivery ratio and minimum control overhead with increase in node speed compared to other two protocols.

PERRA is an on-demand routing protocol which concentrates on the energy efficiency and successful packet delivery. GPSR [5] is a novel routing protocol that makes use of greedy approach for forwarding packets. The routing decisions are taken based on the information about the router's immediate neighbor nodes in the network.A wireless network with 30 nodes moving at a speed of $0.1-20 \mathrm{~m} / \mathrm{s}$ is simulated. Between each mobile nodes there are $8 \mathrm{CBR} / \mathrm{UDP}$ sources generating 8 packets/s.

One of the simulation output variables used are data packet delivery ratio (DPDR) which is the ratio of number of packets successfully delivered to the number packets send by the source. Other one is the control overhead which represents the number of control packets send by the protocols such as HELLO, RREQ, RREP and RERR. The simulation parameters used are given in Table 1.

Simulation results are shown in Fig 1 and Fig 2. The Fig 1 gives the comparison results of LSAR with GPSR and PERRA in terms of DPDR. From the figure it is clear that, all the three protocols performs well in low node speed and low network load. But for higher mobility PERRA is not scalable but others two performs better.

When comparing the protocols in terms of control overhead, it is evident from Fig 2 that while PERRA suffers from higher control overhead for higher node speed, both LSAR and GPSR performs better as they use only the local control packet (HELLO packets)
Table1. Parameters of Simulation

\begin{tabular}{|ll|}
\hline Parameter & Measurement \\
\hline Area of simulation & $870 \mathrm{~m} \times 870 \mathrm{~m}$ \\
Nodes & 30 \\
Node speed & $0.1-20 \mathrm{~m} / \mathrm{s}$ \\
Time of Simulation & $700 \mathrm{~s}$ \\
Traffic Source & 8 \\
Traffic Type & CBR \\
Packet Size & $512 \mathrm{bytes}$ \\
\hline
\end{tabular}

LSAR is more scalable than other two protocols with reduced control overhead.

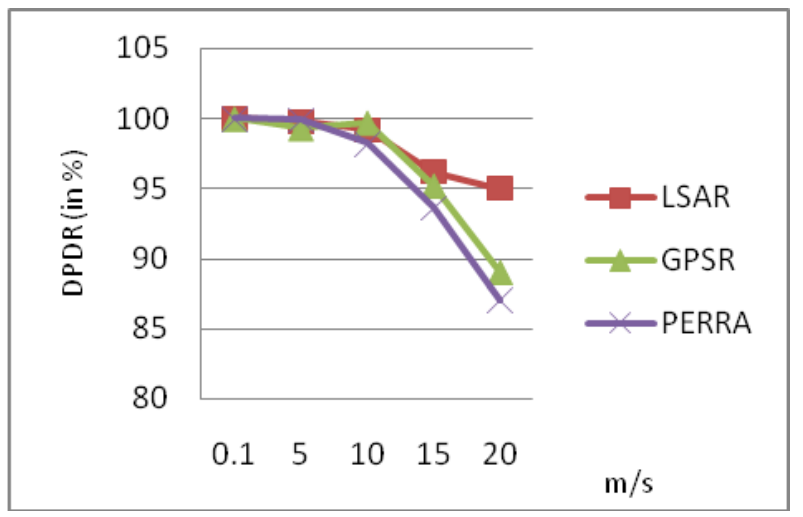

Fig 1. Data Packet Delivery Ratio for different values of node speed

The average link duration for three protocols also are computed and given in Table 2. The link duration increases with a decrease in the node speed. Also higher the mobility of the node, higher will be the link breakage probability.

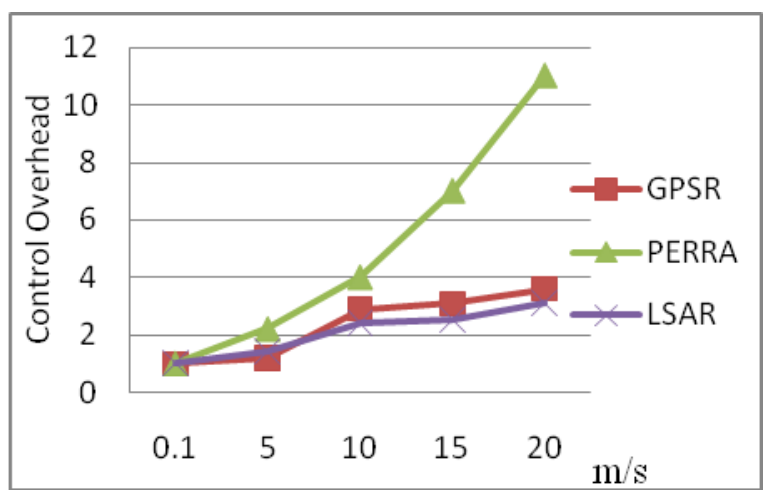

Fig.2. Control overhead Vs Node speed

As the link aware metric is used in all these protocols, the link duration is maintained. However, LSAR protocol considerably increases the link duration by concentrating more on the links history information like its link period. But, PERRA considers only the node speed as criteria for finding a stable link which is not an optimal method from a link stability point of view. 
Table2. Average link duration Versus Node speed

\begin{tabular}{|l|l|l|l|l|l|}
\hline Protocol & $\begin{array}{l}0.1 \\
\mathrm{~m} / \mathrm{s}\end{array}$ & $5 \mathrm{~m} / \mathrm{s}$ & $10 \mathrm{~m} / \mathrm{s}$ & $15 \mathrm{~m} / \mathrm{s}$ & $20 \mathrm{~m} / \mathrm{s}$ \\
\hline PERRA & 110 & 23.5 & 19.5 & 15.1 & 12.6 \\
\hline LSAR & 155 & 28 & 23 & 19 & 14.5 \\
\hline GPSR & 78 & 17.6 & 16.3 & 12.8 & 11 \\
\hline
\end{tabular}

\section{CONCLUSION}

A routing protocol called LSAR has been proposed by considering the link stability metric. QoS is a main concern in routing in MANET. The variation in links quality results in reduced packet delivery ratio, latency and thus affects the QoS. Therefore, incorporating link stability in routing purpose is of importance. LSAR is proposed to find a route with maximum stability by considering the history of links. The performance of LSAR is compared with both GPSR and PERRA and it is shown that LSAR outperforms others in terms of packet delivery ratio and minimum control overhead. Also, the average link duration is higher for LSAR compared to others. We would like to enhance our work by incorporating the energy metric to find a better route that improves network lifetime.

\section{REFERENCES}

[1] B. Karp and H.T. Kung, "Greedy Perimeter Stateless Routing for Wireless Networks," Proc. ACM/IEEE MobiCom '00, pp. 243-254, Aug. 2000.

[2] C.-K-. Toh, "Associativy-Based Routing for Ad-Hoc Mobile Networks," Wireless Personal Comm., vol. 4, no. 2, pp. 103-139, Mar. 1997.

[3] D. Son, A. Helmy, and B. Krishnamachari, "The Effect of Mobility-Induced Location Errors on Geographic Routing in Mobile Ad hoc Sensor Networks: Analysis and Improvement Using Mobility Prediction," IEEE
Trans. Mobile Computing, vol. 3, no. 3, pp. 233-245, July/Aug. 2004.

[4] F. De Rango et al., "A Multi-Objective approach for Energy Consumption and Link Stability Issues in $\mathrm{Ad}$ Hoc Networks," IEEE Comm. Letters, vol. 10, no. 1, pp. 28-30. Jan. 2006.

[5] G. Lim, K. Shin, S. Lee, H. Yoon, and J. Soo Ma, "Link Stability and Route Lifetime in Ad-Hoc Wireless Networks," Proc. Int'l Conf. Parallel Processing Workshops (ICPPW '02), p. 116, 2002.

[6] K. Yamazaki and K. Sezaki, "A Proposal of Geographic Routing Protocols for Location-Aware Services," Electronics and Communications in Japan, vol. 87, no. 4, pp. 26-34, 2003.

[7] Karthikeyan, N. Palanisamy\& V. Duraiswamy, K.A." Performance Evaluation Of Proactive And Reactive Protocols Using ns-2 Simulation," International J. of Engg. Research \& Indu. Appls.(IJERIA). ISSN Pages: 0974-1518 Published: 2009

[8] K.-J. Kim and S.-J. Yoo, "Power-Efficient Reliable Routing Protocol for Mobile Ad-Hoc Networks," IEICE Trans. Comm., vol. E88-B, pp. 4588-4597, 2005.

[9] M. Gerharz, C. de Waal, P. Martini, and P. James, "Strategies for Finding Stable Paths in Mobile Wireless Ad Hoc Networks," Proc.IEEE 28th Ann. Conf. Local Computer Networks (LCN '03), pp. 130-139, 2003.

[10] M. Maleki, K. Dantu, and M. edram, Lifetime Prediction Routing in Mobile Ad Hoc Networks," Proc. IEEE Wireless Comm. and Networking (WCNC '03), pp. 1185 1190, Mar. 2003.

[11] M. Mauve, J. Widmer, and H. Hartenstein, "A Survey on Position-Based Routing in Mobile Ad-Hoc Networks," IEEE Network,vol. 15, no. 6, pp. 30-39. Nov./Dec. 2001.

[12] N. Meghanathan and A. Farago, "Looking at Protocol Efficiency from a New Angle: Stability-Delay Analysis," Proc. Second Int'lConf. Mobile Computing and Networking, pp. 51-55, 2004. 